\title{
338.
}

\section{NOUVELIES RECHERCHES SUR L'ÉLIMINATION ET LA THÉORIE DES COURBES.}

[From the Journal für die reine und angewandte Mathematik (Crelle), tom. LxIII. (1864), pp. 34-39.]

Dans le problème de l'élimination, on cherche la relation qui doit exister entre les coefficients d'une fonction ou système de fonctions pour que quelque circonstance particulière (ou singularité) puisse avoir lieu; par exemple, pour que deux équations puissent avoir une racine commune, ou (comme application géometrique) pour qu'une courbe puisse avoir un point double. En prenant les coefficients comme donnés, tant la relation cherchée que la singularité qu'elle implique n'ont qu'une existence hypothétique. Mais on peut transformer la question en supposant que les coefficients d'une ou de plusieurs des fonctions soient de la forme $a=\lambda a^{\prime}+\mu a^{\prime \prime}, b=\lambda b^{\prime}+\mu b^{\prime \prime}, \ldots$ où $a^{\prime}, b^{\prime}, \ldots$, $a^{\prime \prime}, b^{\prime \prime}, \ldots$ sont des coefficients donnés, mais $\lambda, \mu$ des quantités arbitraires. On peut alors disposer en sorte que la singularité dont il s'agit existe actuellement, en déterminant, au moyen de la relation donnée par l'élimination, la valeur du rapport $\lambda: \mu$. Ces substitutions $a=\lambda a^{\prime}+\mu a^{\prime \prime}, b=\lambda b^{\prime}+\mu b^{\prime \prime}, \ldots$ changent la fonction $U$ à laquelle se rapportent les coefficients $a, b, \ldots$ en $U=\lambda U^{\prime}+\mu U^{\prime \prime}$, où $U^{\prime}, U^{\prime \prime}$ sont des fonctions semblables à $U$, mais avec les coefficients $a^{\prime}, b^{\prime}, \ldots$ ou $a^{\prime \prime}, b^{\prime \prime}, \ldots$ au lieu de $a, b, \ldots$ : en se servant d'une expression usitée, on peut dire que la fonction $U$ est en involution avec $U^{\prime}, U^{\prime \prime}$; et de même en géométrie que la courbe $U=0$ est en involution avec les courbes $U^{\prime}=0, U^{\prime \prime}=0$; au reste, pour les courbes, cela veut dire que les trois courbes se coupent dans les mêmes points.

On conçoit comment cette manière d'envisager le problème peut conduire à une interprétation géométrique de résultats qui n'avaient auparavant qu'une signification analytique. Considérons par exemple la proposition suivante, "le discriminant d'une fonction quadratique à trois variables est du degré 3 par rapport aux coefficients," ou ce qui est la même chose, "la fonction qui égalée à zéro exprime que la conique 
$U=0$ ait un point double (se réduise à une paire de droites) est du degré 3 par rapport aux coefficients," c'est là une proposition purement analytique, mais si comme ci-dessus on met $\lambda a^{\prime}+\mu a^{\prime \prime}, \lambda b^{\prime}+\mu b^{\prime \prime}, \ldots$ au lieu de $a, b, \ldots$ on a le théorème géométrique que voici: "Dans le système de coniques $\lambda U^{\prime}+\mu U^{\prime \prime}=0$ en involution avec les coniques données $U^{\prime}=0, U^{\prime \prime}=0$, il y a 3 coniques à point double (c'est-à-dire, trois paires de droites)." En considérant le cas plus général d'une fonction à trois variables et d'ordre quelconque, la question analytique "quel est le degré du discriminant de la fonction $U$ " peut être remplacée par la question géométrique "dans le système des courbes $\lambda U^{\prime}+\mu U^{\prime \prime}=0$ en involution avec les deux courbes données $U^{\prime}=0, U^{\prime \prime}=0$, quel est le nombre des courbes à point double" ou, ce qui est la même chose, "quel est le nombre des points dont chacun est le point double d'une courbe du système." En considérant la question sous cette dernière forme, non seulement on retrouve la valeur connue $3(n-1)^{2}$ du degré du discriminant de la fonction $U=(A, \ldots \nmid x, y, z)^{n}$, mais on trouve aussi le théorème plus général:

La fonction $U=\left(A, \ldots \chi(x, y, z)^{n}\right.$ étant telle que la courbe $U=0$ ait un nombre a de points doubles et un nombre $\beta$ de points de rebroussement, son discriminant spécial est du degré $3(n-1)^{2}-7 \alpha-11 \beta$.

Sous la désignation de "discriminant special" j’entends la fonction laquelle égalée à zéro donne la condition pour que la courbe $U=0$ ait un point double de plus. Il convient de remarquer par rapport à cette expression que le discriminant de la fonction générale du $n^{\text {ième }}$ ordre, en y substituant, au lieu des valeurs générales, les coefficients de la fonction $U$ dont il s'agit, ne donne nullement le discriminant special de $U$ mais se réduit identiquement à zéro; ce discriminant special est donc tout autre chose que le discriminant de la fonction générale. En parlant tout simplement de l'ordre du discriminant special, j'ai voulu désigner l'ordre auquel cette expression s'élève par rapport à des coefficients absolument arbitraires ou éléments $a, b, \ldots$ lesquels sont censés entrer linéairement dans la fonction $U$. Il est donc nécessaire de démontrer d'abord la proposition auxiliaire que l'équation d'une courbe qui a déjà un nombre donné de points doubles et de rebroussement peut s'exprimer sous la forme signalée, c'est-à-dire linéairement par rapport à des coefficients absolument arbitraires ou éléments $a, b, \ldots$, proposition qui peut être démontrée sans difficulté.

Considérons en effet l'équation générale $U=(A, \ldots \backslash x, y, z)^{n}=0$ où les coefficients $A, \ldots$ sont tous arbitraires; dans le cas d'un point double supposons que les coordonnées de ce point, dans le cas d'un point de rebroussement supposons que les coordonnées de ce point et la direction de la tangente soient données: cela établit pour chaque point double trois conditions, et pour chaque point de rebroussement quatre conditions, qui contiennent d'une manière quelconque les paramètres appartenants au point double ou de rebroussement, mais qui sont linéaires par rapport aux coefficients $A, \ldots$ : ces coefficients peuvent donc s'exprimer linéairement au moyen d'un nombre convenable de coefficients absolument arbitraires ou éléments $a, b, \ldots$; et c'est de ces éléments $a, b, \ldots$ qu'il s'agit et nullement des paramètres mentionnés ci-dessus qui entrent dans les expressions par lesquelles $A, \ldots$ sont donnés en termes de $a, \ldots$. 
Cette proposition auxiliaire peut encore se démontrer de la manière que voici. Concevons que $P=0$ représente une courbe particulière quelconque du même ordre que $U=0$ et telle que pour chaque point double de la courbe $U=0$ elle ait un point double au même point, et que pour chaque point de rebroússement de la courbe $U=0$, elle ait un point de rebroussement au même point et avec la même tangente. Soient de même $Q=0, R=0, \ldots$ des équations de courbes qui satisfont aux mêmes conditions. Cela posé, on peut évidemment écrire $U=a P+b Q+c R+\ldots$, c'est-à-dire que l'équation contiendra linéairement les coefficients absolument arbitraires ou éléments $a, b, \ldots$.

Je reviens au théorème dont je suis parti; soit d'abord $U=\left(a, \ldots \gamma(x, y, z)^{n}=0\right.$ une courbe sans points doubles ou de rebroussement, de sorte qu'il s'agisse du discriminant ordinaire. En écrivant pour plus de simplicité $V, W$ au lieu de $U^{\prime}, U^{\prime \prime}$, on a à considérer la courbe $\lambda V+\mu W=0$ en involution avec les deux courbes $V=0, W=0$. Le degré du discriminant de $U$ est égal au nombre des points dont chacun est le point double d'une courbe particulière du système $\lambda V+\mu W=0$. Or pour trouver ces points on n'a qu'à former les équations

$$
\begin{aligned}
& \lambda \partial_{x} V+\mu \partial_{x} W=0, \\
& \lambda \partial_{y} V+\mu \partial_{y} W=0, \\
& \lambda \partial_{z} V+\mu \partial_{z} W=0,
\end{aligned}
$$

qui expriment que la courbe $\lambda V+\mu W=0$ a un point double, et d'éliminer entre ces équations les indéterminées $\lambda, \mu$. Cela donne le système

$$
\left\|\begin{array}{lll}
\partial_{x} V, & \partial_{y} V, & \partial_{z} V \\
\partial_{x} W, & \partial_{y} W, & \partial_{z} W
\end{array}\right\|=0
$$

qui comprend les deux équations

$$
\left|\begin{array}{ll}
\partial_{x} V, & \partial_{z} V \\
\partial_{x} W, & \partial_{z} W
\end{array}\right|=0
$$

$$
\left|\begin{array}{ll}
\partial_{y} V, & \partial_{z} V \\
\partial_{y} W, & \partial_{z} W
\end{array}\right|=0
$$

auxquelles on satisfait par $\partial_{z} V=0, \partial_{z} W=0$, et une troisièrne équation à laquelle on ne satisfait pas par ce dernier système. Or les courbes (1) et (2) se coupent en $4(n-1)^{2}$ points, mais parmi ceux-ci on a les $(n-1)^{2}$ points d'intersection des courbes $\partial_{z} V=0, \partial_{z} W=0$, et en écartant ces points on obtient $4(n-1)^{2}-(n-1)^{2}=3(n-1)^{2}$ pour le nombre des points, ou ce qui est la même chose pour le degré du discriminant de $U$.

Je suppose à présent que la courbe $U=0$ ait un point double; les courbes $V=0, W=0$ ont chacune un point double à ce même point, et en prenant ce point pour origine des coordonnées $x, y$ les deux courbes seront

$$
\begin{aligned}
& V=z^{n-2}\left(a, b, c \gamma(x, y)^{2}+\text { etc. }=0,\right. \\
& W=z^{n-2}\left(a^{\prime}, b^{\prime}, c^{\prime} \chi x, y\right)^{2}+\text { etc. }=0,
\end{aligned}
$$

en dénotant par les etc. les termes des ordres plus élevés par rapport à $x, y$, ou moins élevés par rapport à $z$. 
Cela donne pour la courbe (1)

$\partial_{x} V, \quad \partial_{z} V \mid=0=z^{2 n-5}\left\{(a x+b y) \cdot\left(a^{\prime}, b^{\prime}, c^{\prime} \gamma(x, y)^{2}-\left(a^{\prime} x+b^{\prime} y\right) \cdot\left(a, b, c \gamma(x, y)^{2}\right\}+\right.\right.$ etc.

$\partial_{x} W, \quad \partial_{z} W \quad=z^{2 n-5} y\left\{(a x+b y)\left(b^{\prime} x+c^{\prime} y\right)-\left(a^{\prime} x+b^{\prime} y\right)(b x+c y)\right\}+$ etc.

la courbe (1) a done à l'origine un point triple, les tangentes étant données par les équations

$$
y=0, \quad(a x+b y)\left(b^{\prime} x+c^{\prime} y\right)-\left(a^{\prime} x+b^{\prime} y\right)(b x+c y)=0,
$$

et de même la courbe (2) a à l'origine un point triple, les tangentes étant données par les équations

$$
x=0, \quad(a x+b y)\left(b^{\prime} x+c^{\prime} y\right)-\left(a^{\prime} x+b^{\prime} y\right)(b x+c y)=0 ;
$$

il y a donc au point triple deux branches de la courbe (1) dont chacune touche une de deux branches de la courbe (2); ce qui donne à l'origine $4+4+3=11$ points d'intersection. De plus il est évident que les deux courbes $\partial_{z} V=0, \partial_{z} W=0$ ont chacune un point double à l'origine, c'est-à-dire elles s'y coupent en $2+2=4$ points.

Par conséquent les courbes (1) et (2) se coupent en $4(n-1)^{2}$ points, savoir

11 points à l'origine, $4(n-1)^{2}-11$ points autrepart, les courbes $\partial_{z} V=0, \partial_{z} W=0$ se coupent en $(n-1)^{2}$ points, savoir

4. points à l'origine, $(n-1)^{2}-4$ points autrepart, et le système des $3(n-1)^{2}$ points contient

7 points à l'origine, $3(n-1)^{2}-7$ points autrepart.

En écartant les points à l'origine on a donc $3(n-1)^{2}-7$ points; pour une courbe à point double le degré du discriminant spécial est donc $=3(n-1)^{2}-7$. Si la courbe $U=0$ a un point de rebroussement, les courbes $V=0, W=0$ auront au même point un point de rebroussement avec la même tangente, et en prenant ce point pour origine des coordonnées et la droite $x=0$ pour l'équation de la tangente, les deux courbes seront

$$
\begin{aligned}
& V=z^{n-2} \cdot a x^{2}+z^{n-3} \cdot\left(\alpha, \beta, \gamma, \delta \gamma(x, y)^{3}+\text { etc. }=0,\right. \\
& W=z^{n-2} \cdot a^{\prime} x^{2}+z^{n-3} \cdot\left(\alpha^{\prime}, \beta^{\prime}, \gamma^{\prime}, \delta^{\prime} \gamma(x, y)^{3}+\text { etc. }=0 .\right.
\end{aligned}
$$

Cela donne pour la courbe (1)

$$
\begin{aligned}
& 0=\left|\begin{array}{ll}
\partial_{x} V, & \partial_{z} V \\
\partial_{x} W, & \partial_{z} W
\end{array}\right| \\
& =\left\{z^{n-2} \cdot 2 a x+z^{n-3} \cdot 3\left(\alpha, \beta, \gamma \gamma(x, y)^{2}+\text { etc. }\right\}\right. \\
& \left.\times\left\{(n-2) z^{n-3} a^{\prime} x^{2}+(n-3) z^{n-4}\left(\alpha^{\prime}, \beta^{\prime}, \gamma^{\prime}, \delta^{\prime}\right\} x, y\right)^{3}+\text { etc. }\right\} \\
& \left.-\left\{z^{n-2} \cdot 2 a^{\prime} x+z^{n-3} \cdot 3\left(\alpha^{\prime}, \beta^{\prime}, \gamma^{\prime}\right\} x, y\right)^{2}+\text { etc. }\right\} \\
& \times\left\{(n-2) z^{n-3} a x^{2}+(n-3) z^{n-4}\left(\alpha, \beta, \gamma, \delta \gamma(x, y)^{3}+\text { etc. }\right\}\right.
\end{aligned}
$$




$$
\begin{aligned}
& =z^{2 n-6}\left\{2 ( n - 3 ) \left[a x\left(\alpha^{\prime}, \beta^{\prime}, \gamma^{\prime}, \delta^{\prime} \gamma x, y\right)^{3}-\alpha^{\prime} x\left(\alpha, \beta, \gamma, \delta \gamma(x, y)^{3}\right]\right.\right. \\
& \left.\qquad-3(n-2)\left[a x^{2}\left(\alpha^{\prime}, \beta^{\prime}, \gamma^{\prime} \gamma x, y\right)^{2}-a^{\prime} x^{2}(\alpha, \beta, \gamma \gamma x, y)^{2}\right]\right\}+ \text { etc. } \\
& =z^{2 n-6}\left\{-n\left(a \alpha^{\prime}-a^{\prime} \alpha\right) x^{4}+\text { etc. } x^{3} y \ldots\right\}+\text { etc. } \\
& =z^{2 n-6} \cdot x\left(-n\left(\alpha \alpha^{\prime}-\alpha^{\prime} \alpha\right), \ldots \gamma(x, y)^{3}+\text { etc. } ;\right.
\end{aligned}
$$

la courbe a donc à l'origine un point quadruple et la droite $x=0 \mathrm{y}$ est tangente de l'une de ses branches. On a de même pour la courbe (2)

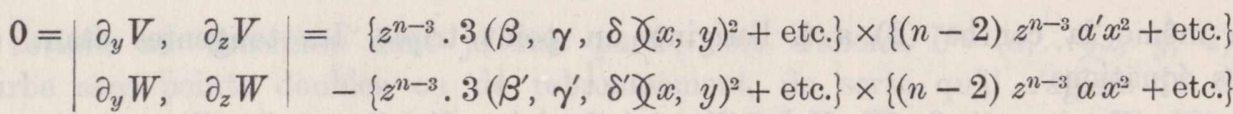

$$
\begin{aligned}
& =z^{2 n-6} x^{2}\left\{a^{\prime}(\beta, \gamma, \delta \gamma x, y)^{2}-a\left(\beta^{\prime}, \gamma^{\prime}, \delta^{\prime} \gamma x, y\right)^{2}\right\}+\text { etc. } \\
& =z^{2 n-6} x^{2}\left(\alpha^{\prime} \beta-\alpha \beta^{\prime}, \ldots \gamma x, y\right)^{2} \text {; }
\end{aligned}
$$

cette courbe a donc à l'origine un point quadruple et la droite $x=0$ y est tangente commune de deux de ses branches. Cela donne à l'origine $5+4+4+4,=17$ points d'intersection des courbes (1) et (2). D'autre part on a

$$
\begin{aligned}
& \partial_{z} V=(n-2) z^{n-3} \cdot a x^{2}+(n-3) z^{n-4}(\alpha, \beta, \gamma, \delta \gamma x, y)^{3}+\text { etc. }=0 \\
& \partial_{z} W=(n-2) z^{n-3} \cdot a^{\prime} x^{2}+(n-3) z^{n-4}\left(\alpha^{\prime}, \beta^{\prime}, \gamma^{\prime}, \delta^{\prime} \gamma(x, y)^{3}+\text { etc. }=0\right.
\end{aligned}
$$

et en combinant ces deux équations

$$
\begin{aligned}
& z^{n-3} \cdot a x^{2}+\text { etc. }=0 \\
& z^{n-4}\left(a \alpha^{\prime}-a^{\prime} \alpha, \ldots \gamma(x, y)^{3}+\text { etc. }=0\right.
\end{aligned}
$$

De ces deux équations la première appartient à une courbe qui a un point de rebroussement à l'origine des coordonnées et la seconde à une courbe qui y a un point triple. Pour les deux courbes $\partial_{z} V=0, \partial_{z} W=0$ cela donne $3+3=6$ points d'intersection à l'origine.

Les courbes (1) et (2) se coupent donc en $4(n-1)^{2}$ points, savoir

17 points à l'origine, $4(n-1)^{2}-17$ points autrepart,

les courbes $\partial_{z} V=0, \partial_{z} W=0$ se coupent en $(n-1)^{2}$ points, savoir

$$
6 \text { points à l'origine, }(n-1)^{2}-6 \text { points autrepart }
$$

et le système des $3(n-1)^{2}$ points contient

$$
11 \text { points à l'origine, } 3(n-1)^{2}-11 \text { points autrepart. }
$$

En écartant les points à l'origine, on obtient $3(n-1)^{2}-11$ points; pour une courbe avec un point de rebroussement, le degré du discriminant spécial est donc $=3(n-1)^{2}-11$.

Comme résultat final de cette recherche j'obtiens que la réduction du degré est de 7 unités pour un point double et de 11 unités pour un point de rebroussement; et 
de là, que pour un nombre $\alpha$ de points doubles et $\beta$ de points de rebroussement, la réduction est de $7 \alpha+11 \beta$ unités; le degré du discriminant spécial sera donc dans ce cas $3(n-1)^{2}-7 \alpha-11 \beta$, ce qu'il s'agissait de démontrer.

Dans tout ce qui précède, j'ai supposé que le système soit tel que l'élimination conduise à une seule relation entre les coefficients; si au contraire l'élimination conduit à deux relations, il faut écrire au lieu de $a, b, \ldots$ les valeurs $\lambda \alpha^{\prime}+\mu \alpha^{\prime \prime}+\nu \alpha^{\prime \prime \prime}, \lambda b^{\prime}+\mu b^{\prime \prime}+\nu b^{\prime \prime \prime}, \ldots$ et de même pour un plus grand nombre de relations. En supposant par exemple que la courbe $U=0$ doit avoir un point de rebroussement, ce qui implique deux relations entre les coefficients, la question à resoudre serait celle-ci, "quel est le nombre des points qui sont chacun un point de rebroussement d'une courbe particulière du système $\lambda V+\mu W+\nu X=0$ "; je réserve à une autre occasion la considération de ce problème.

Londres, 22ième Mai 1863. 